\title{
Dementia of frontal lobe type
}

\author{
D NEARY, J S SNOWDEN, B NORTHEN, P GOULDING
}

From the Department of Neurology, Manchester Royal Infirmary, Manchester, UK

SUMMARY A significant proportion of patients with presenile dementia due to primary cerebral atrophy do not have Alzheimer's disease. One form of non-Alzheimer dementia may be designated as dementia of frontal lobe type (DFT), on the basis of a characteristic neuropsychological picture suggestive of frontal lobe disorder, confirmed by findings on single photon emission tomography. The case histories of seven patients exemplify the disorder: a presentation of social misconduct and personality change, unconcern and disinhibition, in the presence of physical well-being and few neurological signs. Assessment revealed economic and concrete speech with verbal stereotypes, variable memory impairment, and marked abnormalities on tasks sensitive to frontal lobe function. Visuo-spatial disorder was invariably absent. Comparisons of DFT and Alzheimer patients revealed qualitative differences in clinical presentation, neurological signs, profile of psychological disability, electroencephalography, single photon emission tomography and demography. DFT, which may represent forms of Pick's disease, may be more common than is often recognised.

Although Alzheimer's disease represents the commonest form of dementia in the presenium, a significant minority of patients with primary degenerative dementia associated with cerebral atrophy do not have that disease. ${ }^{1-3}$ In particular, patients who present with a clinical picture suggestive of progressive selective damage to frontal regions of the brain have been shown by cerebral biopsy to have neither pathological nor chemical hallmarks of Alzheimer's disease. ${ }^{3}$ The absence of Alzheimer pathology has been confirmed by studies of similar patients at necropsy, ${ }^{4}$ which have indicated the presence of fronto-temporal atrophy, with or without the specific balloon-like cells pathognomonic of Pick's disease, and has led to the designation "dementia of frontal lobe type" (DFT). In clinically presumed cases of DFT the assumption of frontal lobe dysfunction is supported by brain imaging studies using single photon emission tomography (SPET). ${ }^{5}$ The finding in those patients of selective reductions in uptake in the anterior cerebral hemispheres contrasts with the characteristic posterior cerebral hemisphere abnormality in patients with clinically presumed and histologically verified Alzheimer's disease demonstrated both with single photon emission tomography ${ }^{5}$ and positron emission tomography. ${ }^{6-8}$

Address for reprint requests: Dr D Neary, Department of Neurology, Manchester Royal Infirmary, Manchester M13 9WL, UK.

Received 18 August 1987.

Accepted I October 1987
Accurate differentiation between dementing diseases on clinical grounds is increasingly important in view of the possibility of therapeutic intervention. The purpose of this paper is to exemplify, by case studies, the characteristics of DFT, and to distinguish this distinct clinical syndrome from that of Alzheimer's disease by comparing the results of neuropsychological evaluation, SPET imaging and electroencephalography in the two disorders.

\section{Patient group}

Seven patients are described who conformed to a clinical diagnosis of dementia of frontal lobe type. In all patients general medical examination was normal, and history and physical examination excluded the presence of cerebrovascular and systemic disease. No patient had a Hachinski ${ }^{9}$ ischaemia score greater than three. In no patient was there a history of alcohol abuse, or of major head injury. Computed tomographic findings were limited to the presence of mild cerebral atrophy. In some cases (patients 1, 4 and 5) this was reported to be most prominent in the frontal lobes; in other cases no topographical differences in the extent of atrophy were noted. The designation of DFT was based on historical, neurological and neuropsychological findings suggesting a picture of selective frontal lobe dysfunction, and was supported by findings on single photon emission tomographic imaging which revealed selective reductions in uptake in the anterior cerebral hemispheres. ${ }^{5}$ The absence of Alzheimer pathology had been confirmed in two cases by cerebral biopsy.

The patients described are drawn from a series of 15 patients, in whom the clinical picture of frontal lobe dementia was supported by the finding of selective anterior 
hemisphere abnormalities on SPET imaging. They are representative clinically and demographically of a total of 26 patients with a clinical diagnosis of dementia of frontal lobe type, referred to a neurological department during a 5 year period.

\section{A. Case reports}

Patient 1 A 55 year old female presented with a 2 year history of mental slowing, increasing apathy, neglect of personal hygiene and household chores. Occasional incontinence of urine led to no distress, embarrassment nor appropriate explanation. Her mood was reported to be abnormal and variable; sometimes she was irritable, negativistic and uncooperative, and on other occasions jovial and amenable. She denied any change in personality, or mental and physical health. She drove her car with less care, but without becoming lost. She spoke less and more quietly. There was no previous personal or family history of psychiatric illness. Neurological examination revealed extensor plantar responses, a positive jaw jerk and prominent snout reflex. On mental examination she was inert, with a bland affect. She lacked persistence in carrying out mental tasks, although satisfactory performance could often be elicited with coaxing and encouragement. She did not volunteer conversation, and her answers to questions were economical and unelaborated. There was mild hypophonia and dysprosody. She could designate left and right correctly and comprehension of sentences involving complex syntax was intact. Confrontation naming was normal. She could read and spell, although writing yielded striking perseveration: when asked to write about her job, she wrote "I mind about the weather" eight times in succession despite continual encouragement to write something different. In calculating she responded impulsively, making errors. When encouraged to check her responses she could generally make appropriate corrections. Elementary perceptual and spatial abilities were intact. She could identify line drawings of objects and faces of celebrities, could localise correctly towns on a map of Great Britain and reproduce nonrepresentational hand postures accurately. Drawings were normal, and she could represent the three dimensional aspect of a cube. She could reproduce simple Koh's block figures. She could carry out whole body, limb and buccofacial gestures and reproduce alternating hand positions and motor sequences. She was fully oriented for time and place. Her digit span was six digits forwards, and two backwards. She could repeat a seven item name and address immediately after its oral presentation, and recalled 4 of 7 items after a 2 minutes delay. Her retention of a short story was accurate over an interval of one hour, although repeated probing was necessary to elicit the required information.

Standardised psychological testing yielded a WAIS ${ }^{10}$ verbal IQ of 86, performance IQ of 76, and full scale IQ of 81 . On the Warrington recognition test ${ }^{11}$ she scored $32 / 50$ on the word test indicating impaired performance. On the face version she scored $39 / 50$, lying at the 25 th percentile for her age group. She performed poorly on tasks sensitive to frontal lobe dysfunction. In the Nelson modification of the Wisconsin card sorting test ${ }^{12}$ she achieved two sorting categories only. Nineteen per cent of errors were perseverative. In a verbal fluency test she could produce only six animals and four words beginning with the letter $F$, each in one minute. Design fluency ${ }^{13}$ revealed striking perseveration: she produced two pages of virtually identical rectangles, despite constant repetition of the instruction to produce different non-representational designs.

Deterioration in her mental status was observed over a 1 year follow-up period. She became increasingly apathetic, maintained a fatuous expression and giggled inanely when spoken to. Her test performance was characterised by increasing economy of mental effort, although there was no convincing evidence of specific linguistic or perceptuospatial difficulties. On repeat administration of the Nelson card sorting test she achieved one category only, and $56 \%$ of errors were perseverative. She remained physically well, with no increase in neurological signs.

Patient 2 A 61 year old female presented with a 5 year history of progressive change in personality and social conduct. Formerly, she had taken pride in her personal appearance, her home, and her job. She began to neglect personal hygiene, ceased to carry out domestic tasks, and would spend her days wandering aimlessly. She tended to misconstrue events, and expressed suspicion and irritability against her family. She had outbursts of rage which were socially embarrassing. She was forgetful, disclaimed previous events, and made false claims for non-existent happenings. She was hypochondriacally obsessed with pain in the perineum, which had been extensively investigated, but she denied mental symptoms. There was no personal history of psychiatric illness. Her mother had died aged 65 years of a similar illness, and was noted to have wandered in the street at night in night attire. Eccentric behaviour had been observed also in other family members. In no case had necropsy been undertaken. Neurological examination revealed mildly accentuated tendon reflexes and a weakly positive jaw jerk. On mental examination she was restless, impatient and disinhibited. She responded impulsively, launching into tasks without listening fully to the instructions. She displayed a press of speech, which was frequently off the point, and contained stereotyped phrases and play-on-words, which amused her. Her thinking was literal and concrete. In following verbal instructions she had difficulty grasping what was required, although formal assessment of comprehension of syntax did not reveal specific abnormalities. There was some word finding difficulty, and she made occasional verbal paraphasic errors. She could read, and produce a well formed script which was grammatically correct, and could carry out simple written sums, although she made errors on mental subtractions. Elementary perceptual and spatial abilities were intact. Drawings showed preserved spatial configuration, but omission of detail. She could reproduce Koh's block figures, although manipulated blocks in an arbitrary fashion until the correct solution was achieved, apparently with no concerted strategy for carrying out the task. Whilst manual dexterity and manipulative abilities were intact, executive tasks involving production of a sequence of motor actions presented difficulty. She failed to reproduce simple motor rhythms, and a sequence of three hand postures, immediately after these were demonstrated. She was oriented for time and place. She had a normal digit span of six digits forwards and three backwards. She recalled only 4 of 7 items from a name and address immediately after hearing it, and no items after 
a 2 minute delay. She could not abstract the gist of a paragraph read to her.

On the WAIS she achieved a verbal IQ of 73, performance IQ of 77 and full scale IQ of 75. Performance was at chance level on both the word and face section of the Recognition Memory test. On the card sorting test she achieved three categories, from a possible total of eight, and $49 \%$ of errors were perseverative. In a verbal fluency task she produced only eight animal words and seven words beginning with $F$ in one minute. In a design fluency test she violated constantly the two rules that designs should be non-representational and be constructed from four lines only, despite constant reiteration of these instructions. During a 1 year follow-up period change in her condition was minor. However, there was a suggestion of increasing rigidity in her behaviour, more perseverative and stereotyped speech and more florid anomia. She remained over-active and disinhibited.

Patient 3 A 49 year old housewife had progressively altered in her personality and behaviour over 3 years. Previously an out-going, pleasantly mannered and hard-working person, she had become stubborn, lacking in motivation, neglectful of self-care, personal hygiene and domestic responsibilities, and her behaviour was socially embarrassing, inappropriate and disinhibited. She adhered fixedly to a rigid and obsessional daily routine, while spending much of the day pacing restlessly, laughing and chattering inanely. She fabricated tales, often expressing paranoid ideas, and gave distorted accounts of real events. However, she appeared able to retain information of emotional significance to her. Her speech, sight, and ability to find her way were thought to be normal The patient denied mental symptoms, and interpreted her hospital admission as her rejection by her husband. There was no history of previous psychiatric illness. Her eldest brother and a cousin had suffered from a similar illness, dying in their mid-forties and mid-fifties respectively. No necropsy had been performed. At presentation neurological examination was normal. On mental examination, she was unconcerned, mildly disinhibited and distractible. Responses were produced impulsively, without thought, resulting in errors. Her immediate response to questions was often "I don't know", even though with encouragement she could produce the correct answer. Utterances were grammatically correct, although content was not always to the point, and she tended to substitute generic terms such as "thing" for the correct noun, indicating word finding difficulty. She employed words idiosyncratically, for example using "system" as an alternative to "thing". Verbal paraphasias were elicited by confrontation naming testing (for example "bisexuals" for lens of spectacles). She could read, write and spell, although with sporadic errors which suggested attention failure rather than a primary linguistic disorder. She made errors in calculating, although again some variability in her performance was noted. There was no evidence of visual agnosia, spatial disorientation or apraxia. She could identify line drawings and faces of celebrities, showed intact geographical orientation, and could reproduce nonrepresentational hand postures accurately. Gesture and pantomime, and reproduction of drawings and Koh's block figures were all carried out normally. Explanation of pictorial scenes was limited by her unwillingness to apply herself to the task, and tendency to say "I don't know". She was fully oriented and gave a good account of personal day to day events. However, she failed to provide information regarding current news events. Her digit span was six digits forwards, and three backwards. She repeated a seven item name and address correctly, immediately after its presentation, but denied memory for it after a 2 minute delay. Abstraction of the gist of a short paragraph read to her was severely limited immediately after presentation, and she showed no recall after a 1 hour delay. Her performance on both word and face versions of the Warrington recognition memory tests was significantly impaired, her scores lying below the 5 th percentile for her age group.

On the WAIS she achieved a verbal IQ score of 89 , performance score 92 and full scale IQ 90 . On the card sorting test performance was highly abnormal. She achieved one category only, and $100 \%$ of errors were perseverative. She made no attempt to change her method of sorting, despite appreciation that her responses were incorrect. On a verbal fluency test she produced only six animal words and nine $F$ words each in one minute. On a design fluency test she demonstrated perseveration of a single idea, and violated the rule that designs should be constructed from four lines only. Six months after initial examination extensor plantar responses and a positive suck reflex were elicited on neurological examination. A further 6 months later a grasp reflex was present. Her family were aware of a deterioration in her behaviour; she had hecome increasingly restless, inattentive and socially disinhibited, and she would eat and drink greedily and to excess. She would wander the streets for miles, although without becoming lost. On examination, she was restless and distractible. Her verbal output was more restricted than formerly: she did not initiate conversation and she answered questions monosyllabically or with maximum economy of mental effort. Speech was increasingly repetitive, with liberal use of expletives and stereotyped phrases. She tended to "echo" questions put to her in place of constructing a novel response. However, she remained fully oriented, and there was no evidence of visuo-spatial disorder.

Patient 4 At the age of 57 years a previously efficient and independent business woman became progressively more disorganised and irrational, neglecting both her business and domestic responsibilities. She became apathetic, and lacking in motivation, although she denied feelings of depression or anxiety, and appeared to be unaware of ill-health. Her speech and sight were thought to be normal. The patient's father had suffered a similar change in personality and behaviour in his middle years, and spent his last years in a mental hospital. His symptoms at the time had been attributed to heavy alcohol intake. No necropsy had been carried out. On initial examination, 6 years after onset of symptoms, there were no physical signs. Behaviour oscillated between facile and giggling compliance and sullen uncooperativeness. She carried out tasks impulsively without checking, expending minimal mental effort. She was unconcerned by failures. She did not initiate conversation, and responses to questions were brief and unelaborated. Her utterances were linguistically correct and without paraphasic error, although content indicated some concreteness of thought, and some word finding difficulty. She could read, write and spell normally. Elementary visual perception, spatial orientation and praxis were normal. However, she failed to interpret thematic pictorial scenes, itemising elements of the picture 
while making no attempt to integrate elements meaningfully. Drawings and block constructions showed intact spatial configuration, but some omission of detail and poor organisation. She was fully oriented, her digit span was seven digits forwards and three backwards, and immediate recall of a seven item name and address was error-free. However, she failed to recall any information from the name and address after a 2 minute delay, even with the aid of multiple-choice alternatives. Recognition memory performance for both verbal and non-verbal material was at chance level. Her verbal fluency performance was limited to only six animals and five $F$ words in one minute. Two years after initial investigations assessment with the WAIS yielded a verbal IQ of 59, performance IQ of 60 and full scale IQ of 57. Although superficially cooperative, she showed no concern regarding whether her answers were correct, and responses were often fatuous or perseverative. On the Nelson card sorting test she could complete only one sorting category, and $82 \%$ of errors were perseverative. Anomia was noted. Over the following year her mental condition deteriorated. She would spend the day pacing up and down. Her verbal output decreased. She did not speak spontaneously, and responses could be elicited only if questions required a monosyllabic reply. She would giggle inappropriately, and exhibit mannerisms such as humming and foot tapping. She could however find her way about the home and did not appear spatially disoriented. Examination was limited by poor cooperation and negativism. However, there was no evidence of neurological signs, and primary perceptual, spatial and executive abilities appeared intact apart from perseveration elicited by drawing and other motor tasks. Her condition worsened considerably over the ensuing 12 months, necessitating her institutional care. She remained restless, and would pace the ward, aligning objects obsessionally. When seated she exhibited stereotyped rocking trunkal movements, and constant hand clapping, and because of injuries incurred her hands had to be padded. She had become totally dependent, making no attempt to dress or wash herself. She would eat voraciously, although did not mouth inanimate objects. She was virtually mute, although occasional echolalia or use of the word "yes" occurred. Neurological examination revealed the presence of a positive snout reflex and palmomental response. She failed to respond to verbal commands, but could negotiate her way around her environment without difficulty, suggesting preserved perceptuo-spatial appreciation. The SPET scan of the brain revealing reduced uptake selectively in the anterior cerebral hemisphere was carried out at this time. A further 12 months later, there was marked weight loss, and she no longer fed herself. She was doubly incontinent. She had a bland affect, maintained a fatuous, grinning expression and was mute. Neurological findings remained unremarkable.

Patient 5 A 64 year old male had received psychiatric treatment for an unverifiable illness in his mid-twenties. In his late forties, he began to neglect his appearance, became less affectionate, and showed no interest in activities beyond his work. At age 61 he became irritable and intolerant, and then increasingly withdrawn and restless, with poor appetite and insomnia. A diagnosis of agitated depression was made and his condition improved slightly following electroconvulsive therapy. However, he remained apathetic and inactive. His family noted that he displayed virtually no emotion or motivation. Although he was not inclined to converse his actual speech was thought to be normal, and he had no difficulty finding his way about. There was a paucity of information regarding family history. Both parents died in their fifties, although the nature of their terminal illness is unknown. Neurological examination revealed bilateral extensor plantar responses, a prominent snout reflex and a mildly positive palmomental reflex bilaterally. His speech was hypophonic and dysprosodic. On mental examination he was inert, with a bland affect. Motor activity was generally slow, although paradoxically, impulsive responses also occurred. He was cooperative, although made no attempt to respond to tasks of a mentally demanding nature. He did not initiate conversation, and his responses were brief, and often elicited only by continual encouragement. Yet there was no evidence of linguistic disorder, and no paraphasias or word finding difficulty were noted. Comprehension was intact, although interpretation of metaphor and proverb was concrete. He could read, write and spell, but had difficulty calculating, particularly in carrying out mental subtractions. There was no evidence of agnosia, spatial disorientation or apraxia. He could identify line drawings and faces of celebrities, reproduce non-representational hand postures, and use gesture and pantomime accurately. His drawings and block constructions showed preserved spatial configuration, although he showed some lack of persistence, abandoning tasks without checking the accuracy of his attempts. He was fully oriented, had a normal digit span of seven digits forwards and five backwards, and could repeat a seven item name and address accurately. After a 2 minutes delay his recall of the latter was $5 / 7$. He could provide the gist of a short story read to him and showed no loss of information over an interval of one hour. On the Recognition Memory test he scored 43/50 on the word task, which is within normal limits, and $26 / 50$ on the face task, which is at chance level

Assessment with the WAIS yielded a verbal IQ of 73, performance IQ of 80 , and full scale IQ of 74. Performance on the Nelson card sorting test was highly abnormal: he achieved one category only, and $93 \%$ of errors were perseverative. Verbal fluency was reduced: he produced seven animals and seven $F$ words, each in one minute. Design fluency was grossly abnormal: he produced 61 designs in a 10 minute period, 59 of which were perseverative. His clinical picture changed little over a 2 year follow-up period. He remained inert, although with increasing somnolence. He did not initiate conversation nor purposeful activity. Despite paucity of verbal output there was no evidence of aphasia. He could demonstrate retention of day-to-day events, and showed no perceptual or spatial disturbance.

Patient 6 Following a minor concussive head injury at the age of 48 years a 50 year old shop manager suffered a gradually progressive change in his personality. He became outspoken, rude and callous, lacking in initiative, self-care and responsibility towards his family. His mood would fluctuate between facetiousness and jocularity and negativism and irritability, with verbal and physical aggression, thought to be totally out of character. He was inattentive and forgetful, but no change in his speech nor in his vision had been noted. His mother died aged 82 years in a psychiatric hospital, having become demented in her middle fifties. Prior to death she was mute and had remained in an essentially vegetative state for the previous 10 years. No necropsy was undertaken. On examination he was free from neurological 
signs. He was generally courteous, although mildly disinhibited, talking excessively loudly, and giving frequent and inappropriate guffaws of laughter. In carrying out tests he was impulsive and unconcerned, failing to check his responses. He showed no insight. His speech was normal. There was no evidence of impaired comprehension, he was left/right oriented, and no word finding difficulty was evident. Repetition tasks elicited perseverations of previous responses. He could read and write, although spelling was poor. He showed substantial difficulty in calculating, failing to carry out the simplest mental subtraction and completing two figure written sums only by counting on his fingers. He had no difficulty in the perceptual identification of objects, line drawings and faces of celebrities. He could locate objects in the environment, match fingers on the corresponding hands, reproduce non-representational hand postures, and locate towns on a map of Great Britain. He could pantomime actions and use gesture normally. Block and stick constructions were produced slowly, but were accurate copies of the original. Drawings were crude, slapdash and with omission of detail, although spatial relationships between elements were preserved. Perseverative pencil strokes were apparent. He was fully oriented, and displayed preserved memory for day-to-day events. He recalled 6 of 7 items from a name and address immediately following presentation, and again after a 2 minute delay. He could not summarise a short story immediately after reading it aloud, although his ability to provide accurate answers to specific questions about the story indicated that information was available to him. His delayed recall performance, after one hour, provided no evidence of loss of information over time. Performance on the word version of the Recognition test was normal $(48 / 50)$, but on the face version was at chance level $(26 / 50)$.

WAIS testing yielded a verbal IQ of 92 , performance IQ of 82 and full scale IQ of 87 . He failed to cooperate sufficiently to carry out the Nelson card sorting test. On a verbal fluency task he produced 16 animals and one $F$ word, each in one minute. On a design fluency test his responses depicted concrete objects rather than non-representational figures, and perseveration of a single idea was common. Over a 4 year follow-up period his behaviour became increasingly rigid and inflexible, and violent outbursts were more common. He was inert and lacked initiative. Stereotyped mannerisms were increasingly evident. His cognitive performance remained qualitatively similar, although increasing impulsivity, carelessness and lack of attention to the task resulted in reduced performance accuracy. The SPET scan was carried out 5 years after onset of symptoms.

Patient 7 A male electrician was suspended from work at the age of 55 years because of increasingly clownish behaviour, and a reduction in his level of personal and social responsibility. Following a psychiatric referral he resumed work in a more menial capacity, for which he showed no concern. His behaviour became progressively more puerile, fatuous and disinhibited, resulting in his retirement and forcing his wife to assume responsibility for all household and financial affairs. His wife had noted a memory loss although thought this to be selective in his own favour. The patient denied symptoms, and regarded himself as competent to carry out his job. There was no history of previous psychiatric illness, and no known family history of mental illness or dementia. Neurological findings 2 years after onset of symptoms were limited to exaggerated tendon jerks and a right extensor plantar response. On psychological examination he was distractible and restless, with poor engagement and persistence in mental tasks. Behaviour was disinhibited, impulsive and inappropriate, with an exaggerated and melodramatic emotional display. He would break into song, or relate rhymes without prompting. In his verbal remarks he showed a callous unconcern for the feelings of others. His speech was linguistically correct, without paraphasias. However, utterances, produced rapidly, were frequently off the point, and there was a tendency to produce puns and stereotyped phrases. He did not show formal difficulty in comprehension, although his literal interpretation of metaphors and proverbs indicated concreteness of thinking. There was some word finding difficulty for low frequency words. He could read and write, although spelling was erratic. He had difficulty carrying out mental calculations. Perceptual and spatial abilities were intact, as measured by his ability to identify objects, line drawings, and faces of celebrities, to trace the Money road map, to copy non-representational hand postures, to locate towns on a map of Great Britain, and to reproduce Koh's block designs. His copies of drawings of objects and abstract designs showed accurate overall configuration and spatial relationships between individual elements. He could reproduce mirror images of matchstick figures. Manual tasks were all carried out normally; he could use gesture and pantomime, reproduce sequences of hand positions, and motor rhythms. He was fully oriented for time and place, and could give an accurate account of day-to-day events. He reproduced a seven item name and address immediately after hearing it but failed to recall any items after a 2 minute delay.

On the WAIS he achieved a verbal IQ of 93, performance IQ of 106, and full scale IQ of 99. Performance on the Nelson card sorting test was intact: he achieved six complete categories, and $38 \%$ of errors were perseverative. On a verbal fluency test he produced only nine animal words and six $F$ words in one minute. His behaviour was monitored over the following 6 years, during which time progression was slow but clinically evident. He remained disinhibited, distractible and overactive, constantly making puerile remarks intended as jokes. His press of speech increased so that his utterances, although grammatically correct, were barely intelligible. While his speech content was superficially informative, it was only loosely constrained by the question posed. Moreover, he appeared to have an increasingly restricted repertoire of phrases, sentences and anecdotes which, when "triggered", were reiterated on each occasion with virtually identical wording. He produced the reply "I don't speak German" to all but the simplest verbal commands. He had word finding difficulty, and made verbal but not literal paraphasic errors.

During the 6 years of follow-up he retained a digit span of five or six digits forwards, and four backwards. He could read, and write with a well formed script, although spelling was poor. Perceptual and spatial abilities continued to be well preserved. He remained fully oriented in time and place, and capable of some new learning, as evidenced by his ability to recite anecdotes about recent autobiographical episodes. However, his memory appeared patchy and idiosyncratic. He displayed no knowledge of current news events. Motor skills were excellent; he had normal manual dexterity, and 
could carry out alternating hand movements without difficulty. There was no evidence of motor perseveration. He remained physically well and agile, with the emergence of a positive snout and grasp reflex as the only additional neurological signs. A repeat assessment with the WAIS yielded a verbal IQ of 66 , performance IQ of 82 and full scale IQ of 71 . Word and face recognition memory performance was at chance level. On the Nelson card sorting test he achieved one complete category only, and $95 \%$ of errors were perseverative. The SPET scan revealing anterior hemisphere abnormalities was carried out within 6 months of these assessments. He continued to live at home, but attended a day centre where he adhered inflexibly to a rigid routine.

\section{B. Comparisons between dementia of frontal lobe type and Alzheimer's disease}

The following comparisons are based on findings from those 15 patients with a clinical picture of DFT, in whom SPET imaging had been carried out, and 18 Alzheimer patients in whom the diagnosis had been confirmed by cerebral biopsy. In two DFT patients cerebral biopsy had confirmed the absence of Alzheimer pathology and biochemistry. Principal distinctions between DFT and Alzheimer's disease are summarised in the table.

History In DFT patients early features were characterised by social breakdown and personality change with inappropriate affect and lack of concern. Initiative was reduced, patients neglected hygiene and personal responsibilities, their behaviour became rigid, inflexible and socially inappropriate. Their affect was bland or fatuous, and they exhibited a callous loss of sympathy for others. Hypochondriacal, obsessional and paranoid symptoms and a tendency towards gluttony were common. Insight was lost. Restlessness and distractibility were frequently observed. Memory disturbance was reported as variable and idiosyncratic. Speech abnormalities were not prominent early symptoms, although gradual reduction in speech output was sometimes noted as the disease progressed. Symptoms of visuo-spatial disorientation were notably absent even in advanced disease.

Presenting features in Alzheimer patients, in contrast, were of cognitive rather than social breakdown. Social graces were remarkably well preserved in the early stages. Anxiety, distress and querulousness were common. The earliest symptom in most patients was of failing memory. Symptoms suggestive of visuo-spatial disorder occurred early in the disease, and were sometimes the presenting symptom. Difficulty in verbal expression and in word finding, and in reading and writing were common symptoms, particularly as the disease progressed.

Neurological signs Patients with DFT were remarkably free from neurological signs, even in the presence of gross behavioural and cognitive change. Signs were generally limited to the emergence of "frontal" reflexes as the disease progressed. Akinesia, rigidity and myoclonus which were notable features of the Alzheimer patients were absent in patients with DFT.

Electroencephalography In all DFT patients electroencephalography was normal. In all Alzheimer patients moderate slowing of wave forms was present. Comparable findings have been reported by others in comparing Alzheimer dementia and DFT. ${ }^{14,}$ is

Single photon emission tomography All DFT patients exhibited selective reductions in uptake of tracer in the anterior cerebral hemispheres. All patients with proven Alzheimer's disease who underwent imaging by single photon emission tomography, showed reduced uptake in the posterior cerebral hemispheres.

Table Characteristics of Dementia of frontal-type and Alzheimer's disease

\begin{tabular}{|c|c|c|}
\hline & Dementia of frontal lobe type & Alzheimer's disease \\
\hline History & Early personality change and social breakdown & Early amnesia, spatial disorientation and language disorder \\
\hline Family history & History of dementia common & History of dementia rare \\
\hline Physical signs & Primitive reflexes & Rigidity, akinesia, myoclonus \\
\hline EEG & Normal & Abnormal \\
\hline SPET imaging & Anterior hemisphere abnormalities & Posterior hemisphere abnormalities \\
\hline Conduct and affect & $\begin{array}{l}\text { Apathy, unconcern } \\
\text { Inappropriate jocularity } \\
\text { Disinhibition } \\
\text { Distractibility } \\
\text { Loss of social awareness } \\
\text { Loss of emotional empathy } \\
\text { Hypochondriasis } \\
\text { Obsessionality } \\
\text { Gluttony }\end{array}$ & Preserved social awareness \\
\hline Language & $\begin{array}{l}\text { Economical output } \\
\text { Verbal stereotypes } \\
\text { Concrete } \\
\text { Mutism in late stages }\end{array}$ & $\begin{array}{l}\text { Impaired verbal expression } \\
\text { Comprehension, repetition } \\
\text { and naming disorder } \\
\text { Palilalia in late stages }\end{array}$ \\
\hline Spatial abilities & Preserved & Early spatial disorder \\
\hline Memory & Variable memory loss & Consistent amnesia \\
\hline
\end{tabular}




\section{Mental testing}

Conduct In DFT patients, economy of mental effort and unconcern regarding accuracy of responses characterised test performance. Responses were impulsive and tasks were readily abandoned. Yet, correct answers could often be elicited by cajoling and encouragement. Alzheimer patients, in contrast, applied themselves diligently to tasks, expressing concern regarding the accuracy of their responses.

Language Spontaneous conversation was often reduced in DFT patients, responses being brief and unelaborated. In some patients, there was a press of speech, responses being inappropriately constrained by the question. Stereotyped remarks were common, and echoing of questions or reiteration of phrases occurred. Thinking was concrete, verbal paraphasias occurred, and generic terms replaced precise substantives. Failure to grasp instructions and irrelevant responses to questions gave the impression of comprehension impairment, although variability in performance together with demonstrated preserved understanding of complex syntactic sentences and low frequency substantives suggested that the disorder may not be primarily linguistic. Hypophonia and dysprosody were observed in a minority of patients.

In Alzheimer patients increasing reliance on social platitudes with disease progression suggested a decrease in the amount of information available to the patient. Speech was fluent, although utterances begun could often not be completed owing to loss of train of thought. Comprehension was impaired, particularly where a question involved substantial memory load or functional words expressing a spatial relationship. The idiosyncratic word usage and literal thinking noted in DFT patients were not a feature of Alzheimer patients. Palilalia, absent in DFT patients, was a common feature in Alzheimer patients as the disease progressed.

Visuo-spatial abilities and praxis DFT patients could manipulate objects normally, and in dressing showed no difficulty in spatial orientation of articles of clothing. They could localise objects in space and did not become lost in their environment. Drawings and block constructions revealed preservation of spatial configuration and the relationships between elements. However, in carrying out constructional tasks patients showed little concerted strategy or persistence despite awareness of error. Errors were interpreted therefore as secondary to failures of attention and effective strategy, rather than to visuo-spatial disorientation or apraxia. Spatial disability was notably absent even in patients with advanced disease.

In Alzheimer patients spatial disorientation and apraxia were common. Patients had difficulty localising objects in front of them, they became lost in familiar environments, including their own homes, and in dressing they failed to orient clothing correctly. Drawings and block constructions revealed striking loss of spatial configuration and relationship between elements.

Memory DFT patients were typically well-oriented in place and time, and could provide information regarding day to day events. Yet they performed poorly on formal tests of memory. The discrepancy between the clinical impression of well preserved memory and the demonstrated impairment on formal tests suggested that these patients' "amnesia" resulted from a strategic failure to utilise their memory effectively, rather than an inability to acquire and retain new information per se. Relatives' reports of variable and eccentric memory in these patients would seem consistent with this interpretation.

In Alzheimer patients impaired memory was consistent and pervasive. Temporal and spatial disorientation were common and recall of day to day events was severely limited. Impaired performance on formal memory tests was compatible with and corroborated the clinical impression of amnesia.

\section{Demographic features}

The following comparisons of demographic features are based on the total populations of DFT and Alzheimer patients assessed during a comparable 5 year period. A positive diagnosis of DFT or Alzheimer's disease was based on historical, neurological and neuropsychological findings, and supported by findings on electroencephalography. All patients conformed to the clinical picture of DFT or Alzheimer's disease described above. The presence of cerebrovascular or systemic disease had been excluded and no patient had a history of alcohol abuse or major head injury. The exclusive finding of cerebral atrophy on computed tomography supported the general diagnosis of primary degenerative dementia.

Twenty-six patients met the clinical criteria for DFT, and 112 the criteria for Alzheimer's disease.

Age The mean age of onset of symptoms in the DFT population was 54 years (S.D. 6.7 years), range 42-65; median age 55 years. In the population of Alzheimer patients the mean (and median) age of onset was 60 years (S.D. 6.6 years), range 48-77. The difference between the two groups with respect to age of onset of disease was highly significant (Student's unpaired $t$ test $\mathrm{T}=-4.17, \mathrm{p}<0.01$ two-tailed test). Referral bias would account for the overall youthfulness of the two populations, but not the relative youth of the DFT group compared to the Alzheimer group. The data suggest that DFT is a disease predominantly of the presenium, contrasting with Alzheimer's disease, which becomes more prevalent with increasing age.

Sex Of DFT patients, 11 were female and 15 male. This contrasts with 71 female and 41 male Alzheimer patients. The higher incidence of Alzheimer's disease in females compared with males is well-known. ${ }^{16}$ While the relatively small numbers of DFT patients preclude precise inferences regarding sex incidence, the figures suggest that DFT is much less predominantly a disease of females than is Alzheimer's disease.

Family history A history of dementia in a first degree relative was recorded in $46 \%$ of 26 patients with a clinical diagnosis of DFT. By contrast, amongst the population of 112 patients with Alzheimer's disease a positive family history of dementia could be established in only $13 \%$. These findings are consistent with previous reports of a higher familial incidence in non-Alzheimer forms of dementia. ${ }^{117}$

\section{Discussion}

In DFT social breakdown and personality change are prominent. Cognitive impairment appears to be in the realms predominantly of regulation of behaviour: in 
directing and maintaining attention to a task, implementing effective strategies for achieving a solution and checking of responses. Such an interpretation which suggests a disorder predominantly of frontal lobe function is supported by patients' poor performance on tests known to be sensitive to frontal lobe dysfunction, which place demands on powers of abstraction and mental flexibility. In contrast, Alzheimer patients exhibit a picture of amnesia, aphasia, and spatial disorientation with relative preservation of social behaviour, a neuropsychological profile which points to a disorder of the posterior association cortex and limbic system. Dissociations in clinical findings of DFT and Alzheimer patients indicate that purported distinctions are not merely an artifact of disease severity. Indeed, differences between DFT and Alzheimer's disease persist across a broad spectrum of dimensions: demography, history, neurological signs, psychological features, electroencephalography and brain imaging.

DFT is a clinical designation which specifies the presumed major topographical emphasis of pathology, but not pathogenesis. The presence of circumscribed atrophy of the anterior cerebral hemispheres raises the possibility of Pick's disease, and the clinical profile of DFT patients is consistent with descriptions of that disease, which have emphasised the early breakdown in social behaviour and personality change. ${ }^{1618}$ The question arises however as to whether the population of DFT patients conform to a single pathological entity. Within the DFT group, two broad spectrums of behaviour can be identified: the one of slowness, apathy, inertia and aspontaneity, the other of restlessness, overactivity, distractibility and disinhibition. These behaviour patterns cannot be regarded as mutually exclusive, since features of both may occur in the same patient. Nevertheless, patients do tend to fall predominantly into one or other category; amongst the case studies described patients 1 and 5 epitomise the first group, patients 2,3 and 7 the second. The picture of slowness and inertia is associated with such features as perseveration at the level of single actions, and hypophonia. The disinhibited, overactive picture is associated with more evident word finding difficulty and presence of verbal paraphasias, with a relative absence of neurological signs. Such dissociations in symptomatology suggest that dementia of frontal type is not entirely uniform, and raises the possibility of distinct sub-types.

Pathological reports of Pick's disease have recognised distinct sub-groups. In one report, ${ }^{19}$ a distinction was made between a form of disease with almost exclusively cortical pathology and a second form involving also subcortical structures. In another report, ${ }^{20}$ the authors contrasted forms of disease involving respectively predominantly frontal and predominantly temporal pathology. The suggestion within the present patient series of some clinical heterogeneity would be consistent with such purported regional differences in prominence of pathological change. For example, patients presenting a picture of slowness and inertia may correspond to the presence of subcortical pathology. Conversely, the picture of overactive and disinhibited behaviour with striking anomia might be anticipated to be associated with the presence of temporal cortical pathology.

The designation of Pick's disease in this paper has nevertheless been avoided, since it cannot be assumed that these patients meet pathological criteria for that disease. Moreover, the definition of Pick's disease itself remains controversial, ${ }^{1921}$ so that a descriptive term suggesting a major area of dysfunction rather than pathogenesis seems appropriate. Analysis of biopsy tissue and future necropsy studies ought to determine the presence of any specific neuropathological or neurochemical change and resolve the issues of the topographical distribution of pathological change.

Clinical studies of dementia frequently assume that virtually all patients with primary degenerative dementia associated with cortical atrophy will have Alzheimer's disease. In view of this assumption, the ratio of DFT to Alzheimer patients in the present series (almost 1:4) is staggering. Referral bias and regional variations in the prevalence of a strongly familial disorder may have influenced these figures. However, it may also be that the incidence of DFT has generally been underestimated. Traditional reliance on exclusion criteria for the diagnosis of dementia rather than positive diagnostic criteria would serve to submerge distinct forms of dementia.

In the present study inferences regarding the nature of performance breakdown are drawn largely from a qualitative analysis of patients' performance and the nature of errors. That inferred distinctions are valid is borne out by their strong correlation with independent findings, such as those of brain imaging. Diagnostic distinctions between groups appear to lie less in which tasks the patients fail than how they fail, and a neuropsychological evaluation which relies on the interpretation of numerical scores alone is likely to obscure rather than highlight group differences. Scores on the WAIS, for example, taken in isolation, would give rise in DFT patients, as in Alzheimer patients, to the interpretation of "generalised intellectual impairment", and would fail reliably to distinguish the two groups. That patients with impaired function of the "anterior" and "posterior" cerebral hemispheres may achieve comparable scores, yet perform qualitatively differently on a standard psychological test has been demonstrated also by others. ${ }^{22}$ Traditional emphasis in psychological 
assessment on quantitative measurement of performance may have contributed to an underestimate of the incidence of DFT.

Although DFT accounts for only a minority of patients with presenile dementia, the incidence appears sufficiently great to suggest that Alzheimer's disease should not be regarded as the inevitable diagnosis in patients meeting clinical criteria for primary degenerative dementia. The advent of the distinction between cortical and subcortical dementia ${ }^{23}{ }^{24}$ represents an initial step in the clinical distinction between forms of dementia. A further distinction appears warranted: that between the relatively common "posterior" cortical dementia of Alzheimer's disease and the "anterior" cortical dementia described in this paper.

We thank Mr A W I Burjan, Dr D Dodwell, Dr N MacDermott, Dr R A Shields and Dr H J Testa for assistance in patient investigation, and the North West Regional Health Authority for financial support.

\section{References}

1 Sim M, Turner E, Smith WT. Cerebral biopsy in the investigation of presenile dementia. 1. Clinical aspects. Br J Psychiatry 1966;112:119-25.

2 Coblentz JM, Mattis S, Zingesser LH, Kasoff SS, Wisniewski HM, Katzman R. Presenile dementia. Clinical aspects and evaluation of cerebrospinal fluid dynamics. Arch Neurol 1973;29:299-308.

3 Neary D, Snowden JS, Bowen DM, et al. Neuropsychological syndromes in presenile dementia due to cerebral atrophy. $J$ Neurol Neurosurg Psychiatry 1986;49:163-74.

4 Gustafson L, Brun A, Holmkvist AF, Risberg J. Regional cerebral blood flow in degenerative frontal lobe dementia of nonAlzheimer type. J Cereb Blood Flow Metab 1985;5:141-2 (suppl. 1)

5 Neary D, Snowden JS, Shields RA, et al. Single photon emission tomography using ${ }^{99 \mathrm{~m}} \mathrm{Tc}-\mathrm{HM}-\mathrm{PAO}$ in the investigation of dementia. J Neurol Neurosurg Psychiatry 1987;50:1 101-9.

6 Friedland RP, Budinger TF, Ganz E, et al. Regional cerebral metabolic alterations in dementia of the Alzheimer type: positron emission tomography with $\left(18^{\mathrm{F}}\right)$ fluorodeoxyglucose. $J$
Comp Assist Tomog 1983;7:590-8.

7 Foster NL, Chase TN, Fedio P, Patronas NJ, Brooks RA DiChiro G. Alzheimer's disease: focal cortical changes shown by positron emission tomography. Neurology 1983;33:961-5.

8 Foster NL, Chase TN, Mansi L, et al. Cortical abnormalities in Alzheimer's disease. Ann Neurol 1984;16:649-54.

9 Hachinski VC, Iliff LD, Zilhka E, et al. Cerebral blood flow in dementia. Arch Neurol 1975;32:632-7.

10 Wechsler D. The Measurement of Adult Intelligence. Baltimore: Williams and Wilkins 1944.

11 Warrington Recognition Memory Test. NFER-Nelson Publishing Co., Windsor 1984.

12 Nelson HE. A modified card sorting test sensitive to frontal lobe defects. Cortex 1976;12:313-24.

13 Jones-Gotman M, Milner B. Design fluency: the invention of nonsense drawings after focal cortical lesions. Neuropsychologia 1977;15:653-74.

14 Johannesson G, Brun A, Gustafson L, Ingvar DH. EEG in presenile dementia related to cerebral blood flow and autopsy findings. Acta Neurol Scand 1977;56:89-103.

15 Johannesson G, Hagberg B, Gustafson L, Ingvar DH. EEG and cognitive impairment in presenile dementia. Acta Neurol Scand 1979;59:225-40.

16 Lishman WA. Organic Psychiatry Oxford: Blackwell 1978:541-47.

17 Sulkava R, Haltia M, Paetau A, Wikstrom J, Palo J. Accuracy of clinical diagnosis in primary degenerative dementia: correlation with neuropathological findings. J Neurol Neurosurg Psychiatry 1983;46:9-13.

18 Brun A, Gustafson L. Limbic lobe involvement in presenile dementia. Arch Psychiatr Nervankr 1978;226:79-93.

19 Munoz-Garcia D, Ludwin SK. Classic and generalized variants of Pick's disease: a clinicopathological, ultrastructural, and immunocytochemical comparative study. Ann Neurol 1984;16:467-80.

20 Tissot R, Constantinidis J, Richard J. Pick's disease. In: Vinken PJ, Bruyn GW, Klawans HL, Fredericks JAM, eds. Handbook of Clinical Neurology: Neurobehavioural disorders Amsterdam: Elsevier Science Publishers 1985;46:233-46.

21 Kim RC, Collins GH, Parisi JE, Wright AW, Chu YB. Familial dementia of adult onset with pathological findings of a "nonspecific" nature. Brain 1981;104:61-78.

22 Johanson AM, Gustafson L, Risberg J. Behavioural observations during performance of the WAIS block design test related to abnormalities of regional cerebral blood flow in organic dementia. J Clin Exp Neuropsychol 1986;8:201-9.

23 Albert ML, Feldman RG, Willis AL. The "subcortical dementia" of progressive supranuclear palsy. J Neurol Neurosurg Psychiatry 1974;37:121-30.

24 Cummings JL, Benson DF. Subcortical dementia. Review of an emerging concept. Arch Neurol 1984;41:874-9. 